\title{
FINITELY GENERATED GRADED MULTIPLICATION MODULES
}

\author{
NASER ZAMANI \\ Faculty of Science, University of Mohaghegh Ardabili, P.O. Box 179, Ardabil, Iran \\ e-mail:naserzaka@yahoo.com
}

(Received 4 July 2010; revised 8 March 2011; accepted 11 April 2011)

\begin{abstract}
Let $R=\oplus_{i \in \mathbb{Z}} R_{i}$ be a $\mathbb{Z}$-graded ring and $M=\oplus_{i \in \mathbb{Z}} M_{i}$ be a graded $R$-module. Providing some results on graded multiplication modules, some equivalent conditions for which a finitely generated graded $R$-module to be graded multiplication will be given. We define generalised graded multiplication module and determine some of its certain graded prime submodules. It will be shown that any graded submodule of a finitely generated generalised graded multiplication $R$-module $M$ has a kind of primary decomposition. Using this, we give a characterisation of graded primary submodules of $M$. These lead to a kind of characterisation of finitely generated generalised graded multiplication modules.
\end{abstract}

2010 Mathematics Subject Classification. 13A02, 13C05, 13C13, 13 C99.

1. Introduction. Let $R$ be a commutative ring with a non-zero identity and $M$ be a unital $R$-module. Then $M$ is called a multiplication module if any submodule $N$ of $M$ has the form $\mathfrak{a} M$ for some ideal $\mathfrak{a}$ of $R$ [4]. This gives $N=\mathfrak{a} M=\left(N:_{R} M\right) M$, where $\left(N:_{R} M\right)=\{r \in R: r M \subseteq N\}$ is the residual of $N$ by $M$. The $\operatorname{ring} R$ is called a multiplication ring if any ideal of $R$ is a multiplication $R$-module in the above sense [12]. These concepts have attracted the interest of several authors in the last two decades, and have led to more information on the structure of $R$-modules (see for example $[2,3,7,20,21])$. In [9], multiplication modules in the category of $R$-modules graded by a group $G$ were introduced and some interesting results were obtained (see also [8]). Nevertheless, based on the author's knowledge few results are known on this concept. In this paper we deal with the category of graded multiplication modules over a $\mathbb{Z}$-graded ring $R$ and obtain some concerning results. More precisely, among other things, it will be shown that for a graded submodule $N$ of a finitely generated generalised graded multiplication $R$-module $M$, there exists a primary decomposition $N=\bigcap_{\mathfrak{p} \in \mathcal{P}} N(\mathfrak{p})$, where $\mathcal{P}$ denotes the set of all minimal primes of $\left(N:_{R} M\right)$. We also show that for a finitely generated graded multiplication $R$-module $M$, this kind of primary decomposition for each submodule $N$ of $M$, together with other two conditions, gives that $M$ must be a generalised graded multiplication $R$-module. It should be noted that all of the results remain valid if one replaces the grading group $\mathbb{Z}$ with any torsion-free abelian group $G$ having a total order $\leqslant$ compatible with its structure. However, for the convenience we are interested in more concrete abelian group $\mathbb{Z}$.

The organisation of the paper is as follows. In Section 2, we collect some preliminary results on graded multiplication modules. Some characterisations for 
finitely generated graded multiplication modules will be presented (Theorem 2.6). In Section 3, among other things, we give results about certain graded prime submodules of generalised graded multiplication modules, which yields to a kind of primary decomposition theorem (Theorem 3.6), the main theorem of this section. Section 4 concerns graded primary submodules. A kind of characterisation of graded primary submodules is presented (Theorem 4.6). We also give a characterisation of finitely generated generalised graded multiplication modules (Theorem 4.8).

2. Preliminary results. Throughout the rest of this paper, $R=\oplus_{i \in \mathbb{Z}} R_{i}$ is a $\mathbb{Z}$ graded (commutative with identity) ring and $M=\oplus_{i \in \mathbb{Z}} M_{i}$ is a graded $R$-module. If $x \in M_{i}$, we write $\operatorname{deg}(x)=i$ and say that $x$ is a homogeneous element of degree $i$. The set of all homogeneous elements of $R$ (resp. a typical graded $R$-module $X$ ) is denoted by $H(R)$ (resp. $H(X)$ ). For a multiplicative closed subset $S \subseteq H(R)$, the ring of fractions $S^{-1} R$ turns into a graded ring by $\left(S^{-1} R\right)_{i}=\{r / s: r \in H(R), s \in S, i=\operatorname{deg}(r)-\operatorname{deg}(s)\}$, for each $i \in \mathbb{Z}$. Then $S^{-1} M$ is a graded $S^{-1} R$-module in a usual way. In the case that $\mathfrak{p}$ is a graded prime ideal of $R$ and $S=H(R) \backslash \mathfrak{p}$, we denote $S^{-1} R$ and $S^{-1} M$ by $R_{(\mathfrak{p})}$ and $M_{(\mathfrak{p})}$, respectively. A graded ideal $\mathfrak{m}$ is called * maximal if it is maximal in the lattice of all graded ideals of $R$. It is well known that for each graded prime ideal $\mathfrak{p}$ of $R$, the ring $R_{(\mathfrak{p})}$ is a graded ring with unique *maximal ideal $\mathfrak{p} R_{(\mathfrak{p})}$.

Let $N$ be an arbitrary submodule of $M$. Then by $N^{*}$ we mean the graded submodule of $M$ generated by all homogeneous elements $x \in N$. It is clear that $N^{*}$ is the largest graded submodule contained in $N$.

A proper submodule $P$ is called a prime submodule of $M$ if for $r \in R, x \in M$; $r x \in P$ implies $r \in\left(P:_{R} M\right)$ or $x \in P$. It is easily proved that $P$ is a prime submodule if and only if $\left(P:_{R} M\right)$ is a prime ideal of $R$ and $M / P$ is a torsion-free $R /\left(P:_{R} M\right)$-module [16]. For a graded proper submodule $Q$ of $M$, it is well known that $Q$ is primary if and only if for $r \in H(R), x \in H(M) ; r x \in Q$, gives that $r \in \sqrt{Q:_{R} M}$ or $x \in Q[19$, Section 2.13, Lemma 14]. Similar criterion is true for a graded prime submodule. One can see that if $P$ is a prime submodule then $P^{*}$ is also prime. One can also see that for a graded prime submodule $P$ of $M$ if $\left(P:_{R} M\right)$ is an ${ }^{*}$ maximal ideal, then $P$ is prime.

Let $\mathfrak{p}$ be a graded prime ideal of $R$ and $N$ be a graded submodule of $M$. We put

$$
N(\mathfrak{p})=\{x \in M: r x \in N \quad \text { for some } \quad r \in H(R) \backslash \mathfrak{p}\} .
$$

Then $N(\mathfrak{p})$ is a graded submodule of $M$ containing $N$ with the property that for each graded ideal $\mathfrak{b}$ of $R$ with $\mathfrak{b} \cap(H(R) \backslash \mathfrak{p}) \neq \emptyset,(\mathfrak{b} N)(\mathfrak{p})=N(\mathfrak{p})$. In particular we put

$$
\mathbb{O}(\mathfrak{p})=\{x \in M: r x=0 \quad \text { for some } \quad r \in H(R) \backslash \mathfrak{p}\} .
$$

If $\mathbb{O}(\mathfrak{p})=M$, we say that $M$ is graded $\mathfrak{p}$-torsion, and $M$ is called graded $\mathfrak{p}$-cyclic if there exists $r \in H(R) \backslash \mathfrak{p}$ and $x \in H(M)$ such that $r M \subseteq R x$ [9]. If $M$ is finitely generated and $\mathfrak{p}$ is a minimal prime ideal over $\left(N:_{R} M\right)$ (note that such a $\mathfrak{p}$ must be graded, otherwise $\left.\left(N:_{R} M\right) \subseteq \mathfrak{p}^{*} \subset \mathfrak{p}\right)$, then $N(\mathfrak{p})$ is a $\mathfrak{p}$-primary submodule (see Remark (e)).

As it was defined in [9], the graded $R$-module $M$ is called graded multiplication if for any graded submodule $N$ of $M$ there exists a graded ideal $\mathfrak{a}$ of $R$ with $N=\mathfrak{a} M$. The graded ring $R$ is graded multiplication if each graded ideal of $R$ is a graded multiplication $R$-module. We say that $M$ is a generalised graded multiplication module if any proper graded submodule of $M$ is a graded multiplication $R$-module. 
LEMMA 2.1. Let $M$ be a finitely generated graded $R$-module and $\mathfrak{a}$ be a graded ideal of $R$ such that $\mathfrak{a} M=M$. Then there exists $a \in H(\mathfrak{a})$ with $\operatorname{deg}(a)=0$ such that $(1-a) M=0$.

Proof. This is proved using the usual determinant argument with a careful notice to the degree of elements (see also [6, Remark 2.1(i)]).

Theorem 2.2. ([9, Theorem 5.9]) Let $M$ be a graded R-module. Then $M$ is graded multiplication if and only if for any graded prime ideal $\mathfrak{p}, M$ is graded $\mathfrak{p}$-torsion or graded $\mathfrak{p}$-cyclic.

Lemma 2.3. (cf. [1, Corollary 1.7]) A graded R-module $M$ is a graded multiplication $R$-module if and only if

(i) $\bigcap_{\lambda \in \Lambda}\left(\mathfrak{a}_{\lambda} M\right)=\left(\bigcap_{\lambda \in \Lambda}\left[\mathfrak{a}_{\lambda}+\left(0:_{R} M\right)\right]\right) M$ for any non-empty collection of graded ideals $\mathfrak{a}_{\lambda}(\lambda \in \Lambda)$ of $R$, and

(ii) for any graded submodule $N$ of $M$ and any graded ideal $\mathfrak{a}$ of $R$ with $N \subset \mathfrak{a} M$, there exists a graded ideal $\mathfrak{b}$ of $R$ such that $\mathfrak{b} \subset \mathfrak{a}$ and $N \subseteq \mathfrak{b} M$.

For a graded submodule $N$ of $M$ we set ${ }^{*} V(N)=\left\{P \in{ }^{*} \operatorname{spec} M: N \subseteq P\right\}$, where ${ }^{*}$ spec $M$ denotes the set of all graded prime submodules of $M$.

A graded $R$-module $M$ is said to be graded cyclic if there exists $x \in H(M)$ such that $M=R x$.

LEMMA 2.4. Let $k$ be a field and $t$ an indeterminate over $k$. Let $M$ be a graded module over the graded field $S=k\left[t, t^{-1}\right]$ (i.e. each non-zero homogeneous element is invertible) such that for each graded submodules $N, L$ of $M$ there exists a graded submodule $K$ of $M$ with ${ }^{*} V(N) \cup^{*} V(L)={ }^{*} V(K)$. Then $M$ is a graded cyclic module over $S$.

Proof. By [10, Lemma 1.1.1], $M$ is a graded free $S$ module. We show that $M$ has rank one. Suppose the contrary. Note that $(0)$ is the only *maximal ideal of $S$. Hence, any graded proper submodule $P$ of $M$ satisfies $\left(P:_{R} M\right)=0$, and so it is a graded prime submodule. Choose a non-trivial graded prime submodule $P$ of $M$ and let $x \in H(M) \backslash P, 0 \neq p \in H(P)$ with $\operatorname{deg}(x)=\operatorname{deg}(p)$. Then $S x$ and $S(x+p)$ are graded prime submodules of $M$ such that $S x \cap S(x+p)=0 \subseteq P$. By our assumption, there exists a graded submodule $K$ of $M$ such that

$$
{ }^{*} V(S x) \cup{ }^{*} V(S(x+p))={ }^{*} V(K) .
$$

Now we have $S(x) \in{ }^{*} V(S x) \subseteq{ }^{*} V(K)$, so that $K \subseteq S x$. Similarly, $K \subseteq S(x+p)$. Thus, $K \subseteq S x \cap S(x+p)=0$ and we have

$$
{ }^{*} V(S x) \cup{ }^{*} V(S(x+p))={ }^{*} V(0) .
$$

This gives that $S x \subseteq P$ or $S(x+p) \subseteq P$, which is not the case. Therefore, $M$ must be of rank one, as desired.

THEOREM 2.5. ([9, Theorem 5.18]) Let $M$ be a finitely generated graded $R$-module. Then $M$ is graded multiplication if and only if $M_{(\mathfrak{p})}$ is a graded cyclic $R_{(\mathfrak{p})}$-module for each graded prime ideal $\mathfrak{p}$ of $R$, if and only if $M_{(\mathfrak{m})}$ is a graded cyclic $R_{(\mathfrak{m})}$-module for each * maximal ideal $\mathfrak{m}$ of $R$.

The following is a characterisation of finitely generated graded multiplication modules. 
THEOREM 2.6. Let $M$ be a finitely generated graded R-module. The following statements are equivalent:

(i) $M$ is graded multiplication.

(ii) For any graded submodule $N$ of $M$, there exists a graded ideal a of $R$ such that ${ }^{*} V(N)={ }^{*} V(\mathfrak{a} M)$.

(iii) For any graded prime ideal $\mathfrak{p}$ and any graded submodules $U$ and $W$ of $M_{(\mathfrak{p})}$, there exists a graded submodule $X$ of $M_{(\mathfrak{p})}$ such that ${ }^{*} V(U) \cup{ }^{*} V(W)={ }^{*} V(X)$.

(iv) For any graded prime ideal $\mathfrak{p}$ of $R$, there exist at most one graded prime submodule $P$ of $M$ with $\left(P:_{R} M\right)=\mathfrak{p}$.

(v) For any ${ }^{*}$ maximal ideal $\mathfrak{m}$ with $\left(0:_{R} M\right) \subseteq \mathfrak{m}$, the graded $R / \mathfrak{m}-$ module $M / \mathfrak{m} M$ is graded cyclic.

Proof.

(i) $\Rightarrow$ (ii) is clear.

(ii) $\Rightarrow$ (iii) Let $\mathfrak{p}$ be a graded prime ideal of $R$ and let $U, W$ be graded submodules of $M_{(\mathfrak{p})}$. There exist graded submodules $L$ and $K$ of $M$ such that $U=L_{(\mathfrak{p})}$ and $W=K_{(\mathfrak{p})}$. By our assumption (ii), there exists graded ideals $\mathfrak{a}$ and $\mathfrak{b}$ of $R$ such that ${ }^{*} V(L)={ }^{*} V(\mathfrak{a} M)$ and ${ }^{*} V(K)={ }^{*} V(\mathfrak{b} M)$. It is not hard to see that $* V(\mathfrak{a} M) \cup$ ${ }^{*} V(\mathfrak{b} M)={ }^{*} V(J)$, where $J=\mathfrak{a} \mathfrak{b} M$. So ${ }^{*} V(L) \cup * V(K)={ }^{*} V(J)$. Now, as there is a one-to-one correspondence between the set of all graded prime submodules $P$ of $M$ satisfying $\left(P:_{R} M\right) \cap(H(R) \backslash \mathfrak{p})=\emptyset$ and the set of all graded prime submodules of $M_{(\mathfrak{p})}$, given by $P \mapsto P_{(\mathfrak{p})}$, one can easily see that

$$
{ }^{*} V(U) \cup * V(W)={ }^{*} V\left(L_{(\mathfrak{p})}\right) \cup * V\left(K_{(\mathfrak{p})}\right)={ }^{*} V\left(J_{(\mathfrak{p})}\right)
$$

and the result follows.

(iii) $\Rightarrow$ (iv) Let $\mathfrak{p}$ be a graded prime ideal of $R$ and let $P$ be a graded submodule of $M$ with $\left(P:_{R} M\right)=\mathfrak{p}$. Then in view of [5, p. 35], $R_{(\mathfrak{p})} / \mathfrak{p} R_{(\mathfrak{p})}$ is either a field, or else is of the form $k\left[t, t^{-1}\right]$, where $k$ is a field and $t$ is a homogeneous element of positive degree, which is transcendental over $k$. So by Lemma 2.4, $M_{(\mathfrak{p})} /(\mathfrak{p} M)_{(\mathfrak{p})}$ is a graded cyclic module over $R_{(\mathfrak{p})} / \mathfrak{p} R_{(\mathfrak{p})}$. As $(\mathfrak{p} M)_{(\mathfrak{p})} \subseteq P_{(\mathfrak{p})} \subseteq$ $M_{(\mathfrak{p})}$, we conclude that $P_{(\mathfrak{p})}=(\mathfrak{p} M)_{(\mathfrak{p})}$ or $P_{(\mathfrak{p})}=M_{(\mathfrak{p})}$. These respectively gives that $P=P(\mathfrak{p})=(\mathfrak{p} M)(\mathfrak{p})$ or $P=P(\mathfrak{p})=M$. As $P=M$ is not the case, we must have $P=\mathfrak{p} M$.

(iv) $\Rightarrow$ (v) Evidently $\mathfrak{m} M$ is a graded prime submodule of $M$ with $\mathfrak{m}=\left(\mathfrak{m} M:_{R} M\right)$. Now, as $R / \mathfrak{m}$ is either a field or a graded field, by [10, Lemma 1.1.1], $M / \mathfrak{m} M$ is a finite rank-graded free module over $R / \mathfrak{m}$. Let $N$ be a graded submodule of $M$ with $\mathfrak{m} M \subseteq N \subset M$. It follows that $\left(N:_{R} M\right)=\mathfrak{m}$ and so by our hypothesis $N=\mathfrak{m} M$. This gives that $M / \mathfrak{m} M$ is a graded cyclic $R / \mathfrak{m}$-module.

(v) $\Rightarrow$ (i) By Theorem 2.5, we may assume that $\mathfrak{m}$ is the only *maximal ideal of $R$. By our assumption, there exists $x \in H(M) \backslash \mathfrak{m} M$ such that $M=\mathfrak{m} M+$ $R x$. This gives that $M / R x=\mathfrak{m}(M / R x)$. Thus, by Lemma 2.1 , there exists $a \in$ $H(\mathfrak{m})$ with $\operatorname{deg}(a)=0$ such that $(1-a)(M / R x)=0$. It follows that $M$ is a graded $\mathfrak{m}$-torsion. Now for any graded prime ideal $\mathfrak{p} \subseteq \mathfrak{m}$, as $(1-a) \notin \mathfrak{p}, M$ is also a graded $\mathfrak{p}$-torsion. Consequently, by Theorem 2.2, $M$ must be a graded multiplication $R$-module. 
3. A primary decomposition theorem. Let $M$ be a graded module over a graded integral domain $R$. Then the torsion submodule of $M, T(M)=\left\{x \in M:\left(0:_{R} x\right) \neq 0\right\}$, is a graded submodule. In order to see this, let $0 \neq x_{m}+\cdots+x_{n}=x \in T(M)$. We show that all components $x_{i} \in T(M)(m \leq i \leq n)$. There exists $0 \neq a_{p}+\cdots+a_{q}=a \in R$ with $0=a x=\sum_{i+j=r} a_{i} x_{j}$ for $r=m+p, \cdots, n+q$. It follows that $a_{p} x_{m}=0$, and by induction $a_{p}^{i} x_{m+i-1}=0$ for all $i \geq 1$. Thus, $0 \neq a_{p}^{n-m+1} \in\left(0:_{R} x_{j}\right)$ for $m \leq j \leq n$. So $x_{j} \in T(M)$ for $m \leq j \leq n$ as desired.

LEMMA 3.1. Let $R$ be a graded integral domain, which is not a graded field. Let $M$ be a generalised graded multiplication $R$-module. If $M$ is not a torsion module, then it is a torsion-free module.

Proof. Suppose the contrary, $T(M) \neq 0$ and $M / T(M) \neq 0$. As $R$ is not of the form $k\left[t, t^{-1}\right]$, we can find a non-zero proper submodule $N / T(M)$ of $M / T(M)$. Then there exists a non-zero graded ideal $\mathfrak{a}$ of $R$ such that $T(M)=\mathfrak{a} N$. This gives that $N$ is a torsion submodule of $M$ containing $T(M)$. Hence, $N=T(M)$, which is impossible. This completes the proof.

Let $X=\oplus_{i \in \mathbb{Z}} X_{i}, Y=\oplus_{i \in \mathbb{Z}} Y_{i}$ be graded $R$-modules. By a homogeneous homomorphism $f: X \rightarrow Y$ is a homomorphism satisfying $f\left(X_{i}\right) \subseteq Y_{i}$ for all $i \in \mathbb{Z}$.

THEOREM 3.2. ([9, Proposition 5.6]) The ring $R$ is a graded multiplication domain if and only if it is a graded Dedekind domain.

A non-zero unitary graded module $X$ over a commutative graded ring $A$ is called graded uniform if any two non-zero graded submodules of $X$ intersect non-trivially.

LEMMA. 3.3. Let $M$ be a non-zero torsion-free generalised graded multiplication module over the graded integral domain $R$. Then $R$ is a graded Dedekind domain, and $M$ is a graded uniform $R$-module.

Proof. We note that for each $t \in \mathbb{Z}$, the $t$-th shifted module $M(t)=\oplus_{i \in \mathbb{Z}} M_{i+t}$ is a torsion-free generalised graded multiplication module. Let $0 \neq x \in H(M)$ with $a=$ $\operatorname{deg}(x)$. Then the map $R \rightarrow M(a)$ given by $r \rightarrow r x$ is a homogeneous monomorphism of graded $R$-modules. Thus, $R$ is a graded multiplication domain, and by Theorem 3.2, is a graded Dedekind domain.

For the second part, suppose $M$ is not a graded uniform $R$-module. Then we can find non-zero graded submodules $N$ and $L$ of $M$ such that $N \cap L=0$ and $N+L \subset M$. So there exists a non-zero graded ideal $\mathfrak{a}$ of $R$ such that $N=\mathfrak{a}(N+L)$. Thus,

$$
\mathfrak{a} L \subseteq \mathfrak{a}(N+L) \cap L=N \cap L=0,
$$

which is impossible. So $M$ must be a graded uniform $R$-module.

LEMMA 3.4. Let $R$ be a graded integral domain and $M$ be a torsion-free finitely generated generalised graded multiplication $R$-module. Then $M$ is a graded multiplication module.

Proof. By Lemma 3.3, $R$ is a graded Dedekind domain and $M$ is a graded uniform $R$-module. Now let $0 \neq x \in H(M)$. Then $R x \cong R$ as $R$-modules. As $M$ is graded uniform, $M / R x$ is a torsion $R$-module. Thus, as $M$ is finitely generated, we can find $r \in H(R)$ such that $r(M / R x)=0$ and so $r M \subseteq R x$. Let $t=\operatorname{deg}(r)$. Then the map 
$M(-t) \rightarrow R x$ given by $y \rightarrow r y(y \in M)$ is a homogeneous monomorphism of graded $R$-modules. So $M$ is isomorphic to a graded submodule of $R x$, and thus it is isomorphic to a graded ideal of $R$. Hence, $M$ is graded multiplication by Theorem 3.2.

THEOREM 3.5. Let $\mathfrak{m}$ be an *maximal ideal of $R$ and $M$ be a finitely generated generalised graded multiplication module with $\left(0:_{R} M\right) \subseteq \mathfrak{m}$. Let $P$ be a prime submodule of $M$ properly contained in $\mathfrak{m} M$. Then $P=\bigcap_{n \in \mathbb{N}} \mathfrak{m}^{n} M$ and $P=\mathfrak{m} P$.

Proof. By graded version of Nakayama's Lemma we have $\mathfrak{m} M \neq M$ so that $\mathfrak{m} M$ is a graded prime submodule of $M$. Now let $P$ be a graded prime submodule of $M$ such that $P \subset \mathfrak{m} M$. There exists a graded ideal $\mathfrak{a}$ of $R$ with $P=\mathfrak{a m} M$. As $\mathfrak{m} \nsubseteq\left(P:_{R} M\right)$ and $P$ is a prime submodule of $M$, we conclude that $\mathfrak{a} M \subseteq P$ and so $P \subseteq \mathfrak{m} P$. It therefore follows that $P \subseteq \bigcap_{n \in \mathbb{N}} \mathfrak{m}^{n} M$. In order to prove the other inclusion, we note that $M / P$ is a torsion-free finitely generated generalised graded multiplication module over the graded integral domain $R /\left(P:_{R} M\right)$. Thus, by Lemma 3.3, $R /\left(P:_{R} M\right)$ is a graded Dedekind domain, and so is a (graded) Noetherian domain ([18, II.2.1. Theorem $]$ ). Hence, using $[\mathbf{1 7}, 8.10]$, it follows that $\bigcap_{n \in \mathbb{N}}\left[\mathfrak{m} /\left(P:_{R} M\right)\right]^{n}=0$. On the other hand, Lemma 3.4 gives that $M / P$ is also a graded multiplication $R /\left(P:_{R} M\right)$-module. So by Lemma 2.3, we deduce that

$$
\bigcap_{n \in \mathbb{N}} \mathfrak{m}^{n}(M / P)=\left(\bigcap_{n \in \mathbb{N}}\left[\mathfrak{m} /\left(P:_{R} M\right)\right]^{n}\right)(M / P)=0 .
$$

Therefore, $\bigcap_{n \in \mathbb{N}} \mathfrak{m}^{n} M \subseteq P$ and the proof is complete.

REMARKS:

(a) Assume that $\mathfrak{p}$ is a (graded) prime ideal of $R$ and $P$ is a (graded) prime submodule of $M$ such that $\mathfrak{p}=\left(P:_{R} M\right)$. Then $\mathfrak{p} M \subseteq P \subset M$ and $M / P$ is a torsion-free $R / \mathfrak{p}$-module. It follows that $M / \mathfrak{p} M$ is not a torsion $R / \mathfrak{p}$-module.

(b) Let $M$ be a finitely generated generalised graded multiplication $R$-module. Assume that $\mathfrak{p}$ is a graded prime ideal of $R$ that is not *maximal, and $P$ be a graded prime submodule of $M$ such that $\left(P:_{R} M\right)=\mathfrak{p}$. Using (a) together with Lemma 3.1, we see that $\mathfrak{p} M$ is a graded prime submodule of $M$.

(c) Assume that $N$ is a submodule of $M$ such that $\left(N:_{R} M\right)$ is a graded ideal, then $\left(N^{*}:_{R} M\right)=\left(N:_{R} M\right)$. In particular, if $\mathfrak{p}$ is a graded ideal of $R$ and $P$ is a prime submodule with $\left(P:_{R} M\right)=\mathfrak{p}$, then $P^{*}$ is also prime with $\left(P^{*}:_{R} M\right)=\mathfrak{p}$.

(d) Let $\mathfrak{p}$ be a graded prime ideal of $R$. Assume that $M$ is finitely generated and $N$ is a graded submodule of $M$ such that $\left(N:_{R} M\right) \subseteq \mathfrak{p}$. Then using [15, Theorem 3.3] together with (c) we see that there exists a graded prime submodule $P$ of $M$ such that $N \subseteq P$ and $\left(P:_{R} M\right)=\mathfrak{p}$.

(e) Let $M=\left\langle x_{1}, \ldots, x_{n}\right\rangle$ be finitely generated, $N$ a proper submodule of $M$ and $\mathfrak{p}$ be a prime ideal of $R$ minimal over $\left(N:_{R} M\right)$. Then $N(\mathfrak{p})$ is a $\mathfrak{p}$-primary submodule of $M$. In order to see this, let $r \in R, x \in M \backslash N(\mathfrak{p})$ and $r x \in N(\mathfrak{p})$. These, in turn, give that $x / 1 \notin N_{\mathfrak{p}}, r x / 1 \in N(\mathfrak{p})_{\mathfrak{p}}\left(=N_{\mathfrak{p}}\right)$. But, because of $\sqrt{N_{\mathfrak{p}}:_{R_{\mathfrak{p}}} M_{\mathfrak{p}}}=$ $\mathfrak{p} R_{\mathfrak{p}}, N_{\mathfrak{p}}$ is a primary submodule of $M_{\mathfrak{p}}$ and we must have $r / 1 \in \sqrt{N_{\mathfrak{p}}: R_{\mathfrak{p}} M_{\mathfrak{p}}}$. Hence, we can find a positive integer $t$ such that $r^{t} x_{i} / 1 \in N_{\mathfrak{p}}$ for $i=1, \ldots, n$. Therefore, we can find $u \in R \backslash \mathfrak{p}$ with $u r^{t} x_{i} \in N$ and thus $r \in \mathfrak{p}$. Similar argument shows that $\mathfrak{p} \subseteq \sqrt{N(\mathfrak{p}):_{R} M}$. Hence, by [11, p. 386, Ex. 3], $N(\mathfrak{p})$ is a p-primary submodule of $M$. We will call $N(\mathfrak{p})$ the isolated $\mathfrak{p}$-primary component of $N$.

Now we are ready to prove the following theorem. 
THEOREM 3.6. Let $N$ be a graded submodule of a finitely generated generalised graded multiplication $R$-module $M$. Let $\mathcal{P}$ be the set of all minimal prime ideals of $\left(N:_{R} M\right)$. Then

$$
N=\bigcap_{\mathfrak{p} \in \mathcal{P}} N(\mathfrak{p}),
$$

so that $(\dagger)$ is a primary decomposition of $N$.

Proof. By Remark (e) it suffices to prove the equality. We note that all elements of $\mathcal{P}$ are graded. Suppose that $x \in H\left(\bigcap_{\mathfrak{p} \in \mathcal{P}} N(\mathfrak{p})\right) \backslash N$ and look for a contradiction. Let $\mathfrak{m}$ be a minimal (graded) prime ideal over $\left(N:_{R} x\right)$. There exists $\mathfrak{p} \in \mathcal{P}$ such that $\mathfrak{p} \subseteq \mathfrak{m}$. Evidently $\mathfrak{p} \subset \mathfrak{m}$. Now in view of Remark (d) there exists a graded prime submodule $P$ of $M$ such that $N \subseteq P$ and $\left(P:_{R} M\right)=\mathfrak{p}$. We claim that $P=\mathfrak{p} M$.

To this end, we note that $M / P$ is a torsion-free (finitely generated) generalised graded multiplication $R / \mathfrak{p}$-module. So by Lemma $3.3, R / \mathfrak{p}$ is a graded Dedekind domain. Hence, by $[\mathbf{1 8}$, II.2.1. Theorem], $R / \mathfrak{p}$ is a graded Noetherian domain and $\mathfrak{m}$ is an *maximal ideal of $R$. Now by Remark (b) we see that $\mathfrak{p} M$ is a graded prime submodule of $M$. Furthermore, by Lemma $3.4, M / \mathfrak{p} M$ is graded multiplication (also generalised graded multiplication) over $R / \mathfrak{p}$. So applying Theorem 2.6 for $R / \mathfrak{p}$-module $M / \mathfrak{p} M$ we must have $P=\mathfrak{p} M$.

Now in view of $\left[\mathbf{1 4}\right.$, Result 2] and Theorem 3.5, we have $\mathfrak{p} M=\bigcap_{n \in \mathbb{N}} \mathfrak{m}^{n} M$ and $\mathfrak{p} M=\mathfrak{m} \mathfrak{p} M$. As $R / \mathfrak{p}$ as a graded Dedekind domain is a multiplication domain (Theorem 3.6), and $\left[\left(N:_{R} x\right)+\mathfrak{p}\right] / \mathfrak{p} \subseteq \mathfrak{m} / \mathfrak{p}$, there exists a graded ideal $\mathfrak{a}$ of $R$ with $\mathfrak{p} \subseteq \mathfrak{a}$ such that $\left(N:_{R} x\right)+\mathfrak{p}=\mathfrak{a m}+\mathfrak{p}$. If $\mathfrak{a} \subseteq\left(N:_{R} x\right)+\mathfrak{p}$, then we have $\left[\left(N:_{R} x\right)+\mathfrak{p}\right] M=$ $\left[\left(N:_{R} x\right) \mathfrak{m}+\mathfrak{p}\right] M$. This gives that

$$
\begin{aligned}
\left(N:_{R} x\right) M & \subseteq\left[\left(N:_{R} x\right)+\mathfrak{p}\right] M \\
& \subseteq \bigcap_{n \in \mathbb{N}} \mathfrak{m}^{n}\left[\left(N:_{R} x\right)+\mathfrak{p}\right] M+\mathfrak{p} M \subseteq \mathfrak{p} M .
\end{aligned}
$$

Thus, $\left(N:_{R} x\right) \subseteq \mathfrak{p}$ by [14, Result 2], which contradicts the fact that $\mathfrak{m}$ is a minimal prime over $\left(N:_{R} x\right)$.

So we conclude that $\mathfrak{a} \nsubseteq\left(N:_{R} x\right)+\mathfrak{p}$. Thus, $\mathfrak{a} x \nsubseteq N+\mathfrak{p} x$. As $x \in N(\mathfrak{p}), N \subseteq P=$ $\mathfrak{p} M$ and $\mathfrak{p} M$ is a prime submodule with $\left(\mathfrak{p} M:_{R} M\right)=\mathfrak{p}$, we see that $\mathfrak{a} x \subseteq \mathfrak{p} M$. So there exists a graded ideal $\mathfrak{b}$ of $R$ such that $\mathfrak{a} x=\mathfrak{b} \mathfrak{p} M$. This gives that

$$
\begin{aligned}
\mathfrak{a} x & =\mathfrak{b} \mathfrak{p} M=\mathfrak{b} \mathfrak{m} \mathfrak{p} M=\mathfrak{m} \mathfrak{a} x \\
& \subseteq(\mathfrak{m a}+\mathfrak{p}) x=\left[\left(N:_{R} x\right)+\mathfrak{p}\right] x \subseteq N+\mathfrak{p} x,
\end{aligned}
$$

which is a contradiction. The proof is now complete.

\section{Characterisation results.}

Proposition 4.1. Let $M$ be a finitely generated graded R-module. Assume that for each graded submodule $N$ of $M$ we have $N=\bigcap_{\mathfrak{q}} N(\mathfrak{q})$, where $\mathfrak{q}$ runs over all minimal prime ideals of $\left(N:_{R} M\right)$. Suppose that $Q$ is a graded primary submodule of $M$ such that $\left(Q:_{R} M\right)$ is not an * maximal ideal of $R$. Then $Q=\left(Q:_{R} M\right) Q$.

Proof. Set $\mathfrak{q}=\left(Q:_{R} M\right)$ and $\mathfrak{p}=\sqrt{q}$. Suppose that, on the contrary, $Q \neq \mathfrak{q} Q$ and let $x \in H(Q) \backslash \mathfrak{q} Q$. Let $\mathfrak{m}$ be an *maximal ideal of $R$ such that $\mathfrak{q} \subset \mathfrak{m}$. Then $\mathfrak{p}$ is the only minimal prime ideal over $\mathfrak{m}(\mathfrak{q} Q+R x):_{R} M$ and is also the only minimal prime 
ideal over $(\mathfrak{q} Q+R x):_{R} M$. So by our assumption, we have

$$
\begin{aligned}
\mathfrak{m}(\mathfrak{q} Q+R x) & =[\mathfrak{m}(\mathfrak{q} Q+R x)](\mathfrak{p}) \\
& =(\mathfrak{q} Q+R x)(\mathfrak{p})=\mathfrak{q} Q+R x
\end{aligned}
$$

(for the second equality note that $\mathfrak{m} \cap(H(R) \backslash \mathfrak{p}) \neq \varnothing)$. Consequently, there exists $y \in$ $\mathfrak{q} Q$ and $r \in \mathfrak{m}$ such that $x=y+r x$, and so $(1-r) x=y \in \mathfrak{q} Q$. As $\mathfrak{p}$ is the only minimal prime ideal of $\mathfrak{q} Q:_{R} M$, another use of our assumption gives that $\mathfrak{q} Q=(\mathfrak{q} Q)(\mathfrak{p})$ and so $\mathfrak{q} Q$ is a $\mathfrak{p}$-primary submodule of $M$. Therefore, we must have $1-r \in \mathfrak{p} \subset \mathfrak{m}$. Thus, $1 \in \mathfrak{m}$, which is not the case. This completes the proof.

COROLlARY 4.2. Let $M$ be a finitely generated generalised graded multiplication $R$-module. Suppose that $Q$ is a graded primary submodule of $M$ such that $\left(Q:_{R} M\right)$ is not an * maximal ideal of $R$. Then $Q=\left(Q:_{R} M\right) Q$.

Proof. This immediately follows from Theorem 3.6 and Proposition 4.1.

In the next theorem sufficient conditions under which a finitely generated graded multiplication $R$-module to be generalised graded multiplication are given. That these are also necessary conditions, will be given later in Theorem 4.8.

THEOREM 4.3. (cf. $[13,9.21])$ Let $M$ be a finitely generated graded multiplication $R$-module satisfying the following conditions:

(i) For each graded submodule $N$ of $M, N=\bigcap_{\mathfrak{q}} N(\mathfrak{q})$, where $\mathfrak{q}$ runs over all minimal prime ideals of $\left(N:_{R} M\right)$.

(ii) For each graded primary submodule $Q$ of $M$, there exists $n \in \mathbb{N}$ such that $Q=$ $\left(\sqrt{Q:_{R} M}\right)^{n} M$.

(iii) If $N$ is a graded submodule of $M$ and $\mathfrak{p}$ is a minimal prime ideal of $\left(N:_{R} M\right)$, if $n$ is the least positive integer such that $\mathfrak{p}^{n} M=N(\mathfrak{p})$ and if $\mathfrak{p}^{n} M \neq \mathfrak{p}^{n+1} M$, then $\bigcap_{\mathfrak{q}} N(\mathfrak{q}) \nsubseteq \mathfrak{p} M$, where $\mathfrak{q}$ runs over all minimal prime ideals of $\left(N:_{R} M\right)$ other than $\mathfrak{p}$.

Then $M$ is a generalised graded multiplication module.

Proof. Let $N \subseteq L$ be proper graded submodules of $M$. Let $\left\{\mathfrak{p}_{\alpha}\right\}$ be the set of all minimal (graded) prime ideals of both $\left(N:_{R} M\right)$ and $\left(L:_{R} M\right),\left\{\mathfrak{p}_{\beta}^{\prime}\right\}$ be the set of minimal primes over $\left(L:_{R} M\right)$ but not over $\left(N:_{R} M\right)$ and $\left\{\mathfrak{p}^{\prime \prime}{ }_{\gamma}\right\}$ be the set of all minimal primes over $\left(N:_{R} M\right)$ but not over $\left(L:_{R} M\right)$. Then by assumptions (i) and (ii) we have

$$
L=\left(\bigcap \mathfrak{p}_{\alpha}^{h_{\alpha}} M\right) \cap\left(\bigcap \mathfrak{p}_{\beta}^{k_{\beta}} M\right)
$$

and

$$
N=\left(\bigcap \mathfrak{p}_{\alpha}^{m_{\alpha}} M\right) \cap\left(\bigcap \mathfrak{p}_{\gamma}^{\prime \prime n_{\gamma}} M\right)
$$

where $h_{\alpha}, k_{\beta}, m_{\alpha}$ and $n_{\gamma}$ are chosen to be the least positive integers such that $\mathfrak{p}_{\alpha}^{h_{\alpha}} M=$ $L\left(\mathfrak{p}_{\alpha}\right), \mathfrak{p}^{\prime k_{\beta}} M=L\left(\mathfrak{p}_{\beta}^{\prime}\right), \mathfrak{p}_{\alpha}^{m_{\alpha}} M=N\left(\mathfrak{p}_{\alpha}\right)$, and $\mathfrak{p}_{\gamma}^{\prime \prime}{ }^{n_{\gamma}} M=N\left(\mathfrak{p}_{\gamma}\right)$.

For each $\alpha$, we have

$$
\mathfrak{p}_{\alpha}^{m_{\alpha}} M=N\left(\mathfrak{p}_{\alpha}\right) \subseteq L\left(\mathfrak{p}_{\alpha}\right)=\mathfrak{p}_{\alpha}^{h_{\alpha}} M
$$


so by the minimality of $h_{\alpha}$ we have $h_{\alpha} \leq m_{\alpha}$. Set

$$
\mathfrak{c}:=\left(\bigcap \mathfrak{p}_{\alpha}^{m_{\alpha}-h_{\alpha}}\right) \cap\left(\bigcap \mathfrak{p}_{\gamma}^{\prime \prime n_{\gamma}}\right) \text {. }
$$

Then in view of Lemma 2.3 (i), we have $N \subseteq \mathfrak{c} M$. It is not hard to see that $\mathfrak{c} L \subseteq N$. We will prove that $\mathrm{c} L=N$.

Let $\mathfrak{p}$ be a minimal prime ideal over $\left(\mathfrak{c} L:_{R} M\right)$. Then $\left(L:_{R} M\right) \subseteq \mathfrak{p}$ or else $\mathfrak{c} \subseteq \mathfrak{p}$. If $\left(L:_{R} M\right) \subseteq \mathfrak{p}$, then $\mathfrak{p}$ is a minimal prime ideal over $\left(L:_{R} M\right)$. Since $\left(N:_{R} M\right) \subseteq \mathfrak{p}$, there exists a minimal (graded) prime ideal $\mathfrak{q}$ over $\left(N:_{R} M\right)$ such that $\mathfrak{q} \subseteq \mathfrak{p}$. Hence,

$$
\left(\mathfrak{c} L:_{R} M\right) \subseteq\left(N:_{R} M\right) \subseteq \mathfrak{q} \subseteq \mathfrak{p},
$$

and so $\mathfrak{p}$ must be a minimal prime ideal over $\left(N:_{R} M\right)$. Thus, $\mathfrak{p}=\mathfrak{p}_{\alpha}$ for some $\alpha$. On the other hand if $\left(L:_{R} M\right) \nsubseteq \mathfrak{p}$, then $\mathfrak{c} \subseteq \mathfrak{p}$ and it can be easily seen that $\mathfrak{p}$ is a minimal prime ideal over both $\left(N:_{R} M\right)$ and $\mathfrak{c}$. Thus, $\mathfrak{p}=\mathfrak{p}_{\gamma}^{\prime \prime}$. Consequently by (i) we have

$$
\mathfrak{c} L=\left(\bigcap \mathfrak{p}_{\alpha}^{r_{\alpha}} M\right) \cap\left(\bigcap \mathfrak{p}_{\gamma}^{\prime \prime s_{\gamma}} M\right),
$$

where $r_{\alpha}$ and $s_{\gamma}$ are the least positive integers such that $\mathfrak{p}_{\alpha}^{r_{\alpha}} M=(\mathfrak{c} L)\left(\mathfrak{p}_{\alpha}\right)$ and $\mathfrak{p}_{\gamma}^{\prime \prime s_{\gamma}} M=$ $(\mathfrak{c} L)\left(\mathfrak{p}_{\gamma}^{\prime \prime}\right)$.

Since $\mathfrak{c} L \subseteq N$, we have

$$
\mathfrak{p}_{\alpha}^{r_{\alpha}} M=(\mathfrak{c} L)\left(\mathfrak{p}_{\alpha}\right) \subseteq N\left(\mathfrak{p}_{\alpha}\right)=\mathfrak{p}_{\alpha}^{m_{\alpha}} M
$$

Hence, by the minimality of $r_{\alpha}$ we have $m_{\alpha} \leq r_{\alpha}$ for all $\alpha$. Similarly $n_{\gamma} \leq s_{\gamma}$ for all $\gamma$. Now for each $\gamma$ we have

$$
N \subseteq \mathfrak{c} M \subseteq \mathfrak{p}_{\gamma}^{\prime \prime n_{\gamma}} M=N\left(\mathfrak{p}^{\prime \prime}{ }_{\gamma}\right)
$$

Thus,

$$
N\left(\mathfrak{p}^{\prime \prime}{ }_{\gamma}\right) \subseteq(\mathfrak{c} M)\left(\mathfrak{p}^{\prime \prime}{ }_{\gamma}\right) \subseteq\left(\mathfrak{p}^{\prime \prime}{ }_{\gamma}{ }_{\gamma} M\right)\left(\mathfrak{p}^{\prime \prime}{ }_{\gamma}\right)=\left[N\left(\mathfrak{p}^{\prime \prime}{ }_{\gamma}\right)\right]\left(\mathfrak{p}^{\prime \prime}{ }_{\gamma}\right)=N\left(\mathfrak{p}^{\prime \prime}{ }_{\gamma}\right)
$$

which gives that

$$
(\mathfrak{c} M)\left(\mathfrak{p}^{\prime \prime}{ }_{\gamma}\right)=\mathfrak{p}_{\gamma}^{\prime \prime n_{\gamma}} M .
$$

On the other hand, as $L \nsubseteq \mathfrak{p}_{\gamma}^{\prime \prime} M$ or equivalently as $\left(L:_{R} M\right) \nsubseteq \mathfrak{p}_{\gamma}^{\prime \prime}$ (note that $M$ is a graded multiplication), we deduce that

$$
(\mathfrak{c} L)\left(\mathfrak{p}_{\gamma}^{\prime \prime}\right)=\left[\mathfrak{c}\left(L:_{R} M\right) M\right]\left(\mathfrak{p}_{\gamma}^{\prime \prime}\right)=(\mathfrak{c} M)\left(\mathfrak{p}_{\gamma}^{\prime \prime}\right)=\mathfrak{p}_{\gamma}^{\prime \prime n_{\gamma}} M,
$$

which means that $\mathfrak{p}_{\gamma}^{\prime \prime n_{\gamma}} M$ is the isolated $\mathfrak{p}_{\gamma}^{\prime \prime}$-primary component of $\mathfrak{c} L$. So we must have $n_{\gamma}=s_{\gamma}$.

If $\mathfrak{p}_{\alpha}^{m_{\alpha}} M=\mathfrak{p}_{\alpha}^{m_{\alpha}+1} M$, then $\mathfrak{p}_{\alpha}^{m_{\alpha}} M=\mathfrak{p}_{\alpha}^{r_{\alpha}} M$, and by the minimality of $r_{\alpha}$ we conclude that $m_{\alpha}=r_{\alpha}$.

Therefore, suppose $\mathfrak{p}_{\alpha}^{m_{\alpha}} M \neq \mathfrak{p}_{\alpha}^{m_{\alpha}+1} M$. Let

$$
L^{\prime}=\left(\bigcap_{\delta \neq \alpha} \mathfrak{p}_{\delta}^{h_{\delta}} M\right) \cap\left(\bigcap \mathfrak{p}_{\beta}^{\prime k_{\beta}} M\right),
$$




$$
N^{\prime}=\left(\bigcap_{\delta \neq \alpha} \mathfrak{p}_{\delta}^{m_{\delta}} M\right) \cap\left(\bigcap \mathfrak{p}_{\gamma}^{\prime n_{\gamma}} M\right)
$$

and

$$
\mathfrak{c}^{\prime}=\left(\bigcap_{\delta \neq \alpha} \mathfrak{p}_{\delta}^{m_{\delta}-h_{\delta}}\right) \cap\left(\bigcap \begin{array}{c}
\mathfrak{p}^{\prime \prime} n_{\gamma} \\
\gamma
\end{array}\right) .
$$

By condition (iii) $N^{\prime} \nsubseteq \mathfrak{p}_{\alpha} M$. Applying Proposition 4.1 for $\mathfrak{p}_{\alpha} M$, gives that $\mathfrak{p}_{\alpha}$ is an *maximal ideal of $R$. So $R=\mathfrak{p}_{\alpha}^{m_{\alpha}}+\left(N^{\prime}:_{R} M\right)$. This gives that

$$
N=\mathfrak{p}_{\alpha}^{m_{\alpha}} M \cap N^{\prime}=\left(\mathfrak{p}_{\alpha}^{m_{\alpha}} M \cap N^{\prime}\right)\left(\mathfrak{p}_{\alpha}^{m_{\alpha}}+\left(N^{\prime}:_{R} M\right)\right) \subseteq \mathfrak{p}_{\alpha}^{m_{\alpha}} N^{\prime},
$$

and so $\mathfrak{p}_{\alpha}^{m_{\alpha}} M \cap N^{\prime}=\mathfrak{p}_{\alpha}^{m_{\alpha}} N^{\prime}$.

As $h_{\alpha} \leq m_{\alpha}$, we have $\mathfrak{p}_{\alpha}^{h_{\alpha}} M \neq \mathfrak{p}_{\alpha}^{h_{\alpha}+1} M$. Thus, by (iii) we have $L^{\prime} \nsubseteq \mathfrak{p}_{\alpha} M$ or equivalently $\left(L^{\prime}:_{R} M\right) \nsubseteq \mathfrak{p}_{\alpha}$. Therefore, $R=\mathfrak{p}_{\alpha}^{h_{\alpha}}+\left(L^{\prime}:_{R} M\right)$. This gives that

$$
L=\mathfrak{p}_{\alpha}^{h_{\alpha}} M \cap L^{\prime}=\mathfrak{p}_{\alpha}^{h_{\alpha}} L^{\prime}
$$

As $N^{\prime} \subseteq \mathfrak{c}^{\prime} M$ and $N^{\prime} \nsubseteq \mathfrak{p}_{\alpha} M$, we must have $\mathfrak{c}^{\prime} \nsubseteq \mathfrak{p}_{\alpha}$. Consequently if $m_{\alpha}-h_{\alpha}>0$, then $\mathfrak{c}=\mathfrak{p}_{\alpha}^{m_{\alpha}-h_{\alpha}} \cap \mathfrak{c}^{\prime}=\mathfrak{p}_{\alpha}^{m_{\alpha}-h_{\alpha}} \mathfrak{c}^{\prime}$. Hence, in this case we have the equality $\mathfrak{c} L=\mathfrak{p}_{\alpha}^{m_{\alpha}} \mathfrak{c}^{\prime} L^{\prime}$. If $m_{\alpha}-h_{\alpha}=0$, then $\mathfrak{c}=\mathfrak{c}^{\prime}$ and the same equality holds. As $\mathfrak{c}^{\prime}\left(L^{\prime}:_{R} M\right) \nsubseteq \mathfrak{p}_{\alpha}$, thus

$$
(\mathfrak{c} L)\left(\mathfrak{p}_{\alpha}\right)=\left(\mathfrak{p}_{\alpha}^{\mathfrak{m}_{\alpha}} \mathfrak{c}^{\prime} L^{\prime}\right)\left(\mathfrak{p}_{\alpha}\right)=\left[\mathfrak{p}_{\alpha}^{m_{\alpha}} \mathfrak{c}^{\prime}\left(L^{\prime}:_{R} M\right) M\right]\left(\mathfrak{p}_{\alpha}\right)=\left(\mathfrak{p}_{\alpha}^{m_{\alpha}} M\right)\left(\mathfrak{p}_{\alpha}\right)=\mathfrak{p}_{\alpha}^{m_{\alpha}} M,
$$

which means $\mathfrak{p}_{\alpha}^{m_{\alpha}} M$ is the isolated $\mathfrak{p}_{\alpha}$-primary component of $\mathfrak{c} L$. As $m_{\alpha} \leq r_{\alpha}$, it follows that $m_{\alpha}=r_{\alpha}$. Hence, by our assumption (i), ( $\dagger$ ) and ( $\ddagger$ ) we must have $N=\mathfrak{c} L$ as desired.

LemmA 4.4. Let $\mathfrak{m}$ be an *maximal ideal of $R$ and $M$ be a generalised graded multiplication module with $\mathfrak{m} M \neq M$. Then for each $n \in \mathbb{N}$, there is no graded submodule of $M$ properly between $\mathfrak{m}^{n} M$ and $\mathfrak{m}^{n+1} M$.

Proof. Let $N$ be a graded submodule of $M$ such that $\mathfrak{m}^{n+1} M \subset N \subseteq \mathfrak{m}^{n} M$. We show that $N=\mathfrak{m}^{n} M$. There exists a graded ideal $\mathfrak{a}$ of $R$ such that $N=\mathfrak{a m}^{n} M$ and $\mathfrak{a} \nsubseteq \mathfrak{m}$. Hence, $\mathfrak{a}+\mathfrak{m}=R$, and we have $\mathfrak{m}^{n} M=\mathfrak{m}^{n}(\mathfrak{a}+\mathfrak{m}) M=N$ as desired.

LemMA 4.5. Suppose that $\mathfrak{m}$ is an *maximal ideal of $R$ and $M$ is a generalised graded multiplication $R$-module. Let $\mathfrak{m}^{n} M \neq \mathfrak{m}^{n+1} M$ for each $n \in \mathbb{N}$. Then $P:=\bigcap_{n \in \mathbb{N}} \mathfrak{m}^{n} M$ is a graded prime submodule of $M$.

Proof. Clearly $P$ is a proper graded submodule of $M$. Let $x \in H(M) \backslash P$ and $r \in H(R) \backslash\left(P:_{R} M\right)$. Then there exists $i, j \geq 0$ such that $x \in \mathfrak{m}^{i} M \backslash \mathfrak{m}^{i+1} M$, and $r \in$ $\left(\mathfrak{m}^{j} M:_{R} M\right) \backslash\left(\mathfrak{m}^{j+1} M:_{R} M\right)$. This shows that there exists $y \in H(M)$ such that $r y \in$ $\mathfrak{m}^{j} M \backslash \mathfrak{m}^{j+1} M$. Therefore, by Lemma 4.4 we have

$$
\mathfrak{m}^{i} M=\mathfrak{m}^{i+1} M+R x
$$

and

$$
\mathfrak{m}^{j} M=\mathfrak{m}^{j+1} M+R(r y) .
$$


Thus,

$$
\begin{aligned}
\mathfrak{m}^{i+j} M= & \mathfrak{m}^{i}\left(\mathfrak{m}^{j+1} M+R(r y)\right)=\mathfrak{m}^{i+j+1} M+\mathfrak{m}^{i}(r y) \\
& \subseteq \mathfrak{m}^{i+j+1} M+r \mathfrak{m}^{i+1} M+R(r x) \subseteq \mathfrak{m}^{i+j+1} M+R(r x) .
\end{aligned}
$$

As by our assumption $\mathfrak{m}^{i+j} M \nsubseteq \mathfrak{m}^{i+j+1} M$, we deduce that $r x \notin \mathfrak{m}^{i+j+1} M$. So $r x \notin P$ and $P$ must be a graded prime submodule of $M$.

Now we prove the following characterisation of graded primary submodules of a finitely generated generalised graded multiplication $R$-module.

THEOREM 4.6. Let $M$ be a finitely generated graded generalised multiplication $R$ module. Let $Q$ be a graded primary submodule of $M$. Then there exists a positive integer $t$ such that $Q=\left(\sqrt{Q:_{R} M}\right)^{t} M$.

Proof. Put $\mathfrak{p}=\sqrt{Q:_{R} M}$. Then $\mathfrak{p} \neq R$ is a graded prime ideal of $R$. We consider two cases. Case (i): $\mathfrak{p}$ is not an *maximal ideal of $R$.

By Corollary 4.2, $Q=\left(Q:_{R} M\right) Q \subseteq \mathfrak{p} M$. We shall show that $Q=\mathfrak{p} M$. Suppose that $x \in H(\mathfrak{p} M) \backslash Q$. Let $\mathfrak{m}$ be an *maximal ideal of $R$ containing $\mathfrak{p}$ and put $N:=$ $Q+\mathfrak{m} x$. Then, evidently $x \notin N$, and so by Theorem 3.6 there exists a minimal prime ideal $\mathfrak{q}$ over $\left(N:_{R} M\right)$ such that $x \notin N(\mathfrak{q})$. Now let $r \in \mathfrak{p}$. Then using Corollary 4.2, we have $r^{n} M \subseteq Q \subseteq \mathfrak{q} M$ for some positive integer $n$. Therefore, in view of [14, Result 2], we have $r \in \mathfrak{q}$. Hence, $\mathfrak{p} \subseteq \mathfrak{q}$. On the other hand, let $s \in H(\mathfrak{m}) \backslash \mathfrak{p}$. Then $s x \in N \subseteq N(\mathfrak{q})$, which gives that $s \in \mathfrak{q}$ (otherwise $x \in N(\mathfrak{q}$ ), which is not the case). Hence, $\mathfrak{p} \subset \mathfrak{q}$, which contradicts the fact that $\mathfrak{q}$ is a minimal prime over $\left(N:_{R} M\right)$. Therefore, we must have $Q=\mathfrak{p} M$.

Case (ii): $\mathfrak{p}$ is an ${ }^{*}$ maximal ideal of $R$.

First suppose that $\mathfrak{p}^{n} M \neq \mathfrak{p}^{n+1} M$ for each positive integer $n$. Then by Lemmas 3.5 and 4.5 , the submodule $\bigcap_{n \in \mathbb{N}} \mathfrak{p}^{n} M$ is the (only) graded prime submodule of $M$ properly contained in the graded prime submodule $\mathfrak{p} M$.

Suppose $Q \subseteq \mathfrak{p}^{n} M$ for each $n \in \mathbb{N}$. Then $Q \subseteq \bigcap_{n \in \mathbb{N}} \mathfrak{p}^{n} M \subset \mathfrak{p} M$ and so

$$
\begin{aligned}
\mathfrak{p} & =\sqrt{Q:_{R} M} \subseteq \sqrt{\left(\bigcap_{n \in \mathbb{N}} \mathfrak{p}^{n} M\right):_{R} M} \\
& =\left(\bigcap_{n \in \mathbb{N}} \mathfrak{p}^{n} M\right):_{R} M=\bigcap_{n \in \mathbb{N}}\left(\mathfrak{p}^{n} M:_{R} M\right) .
\end{aligned}
$$

Therefore, we have

$$
\mathfrak{p} M \subseteq\left[\bigcap_{n \in \mathbb{N}}\left(\mathfrak{p}^{n} M:_{R} M\right)\right] M \subseteq \bigcap_{n \in \mathbb{N}}\left[\left(\mathfrak{p}^{n} M:_{R} M\right) M\right] \subseteq \bigcap_{n \in \mathbb{N}} \mathfrak{p}^{n} M,
$$

which is not the case. Thus, there exists a positive integer $t$ such that $Q \subseteq \mathfrak{p}^{t} M$ and $Q \nsubseteq \mathfrak{p}^{t+1} M$. Now there exists a graded ideal $\mathfrak{a}$ of $R$ such that $Q=\mathfrak{a p}^{t} M$ and $\mathfrak{a} \nsubseteq \mathfrak{p}$. We claim that $\mathfrak{a}=R$. Suppose the contrary. Let $\mathfrak{n}$ be any *maximal ideal of $R$ with $\mathfrak{a} \subseteq \mathfrak{n}$. Then $\left(Q:_{R} M\right)=\left(\mathfrak{a p}^{t} M:_{R} M\right) \subseteq\left(\mathfrak{n} M:_{R} M\right)=\mathfrak{n}$ and so $\mathfrak{p}=\mathfrak{n}$. As this is impossible, we must have $\mathfrak{a}=R$ and $Q=\mathfrak{p}^{t} M$.

Continuing to assume that $\mathfrak{p}$ is a *maximal ideal of $R$, we suppose that $\mathfrak{p}^{n} M=$ $\mathfrak{p}^{n+1} M$ for some positive integer $n$. Suppose further that $Q \subseteq \mathfrak{p}^{n} M$. If $x \in H\left(\mathfrak{p}^{n} M\right)$, 
then there is a graded ideal $\mathfrak{b}$ of $R$ such that $R x=\mathfrak{b} \mathfrak{p}^{n} M=\mathfrak{b p}^{2 n} M=\mathfrak{p}^{n} x$. Hence, there exists $p \in \mathfrak{p}$ such that $x=p x=p^{2} x=\cdots$, and consequently $x \in Q$ (note that some power of $p$ is in $\left.\left(Q:_{R} M\right)\right)$. Therefore, $Q=\mathfrak{p}^{n} M$. On the other hand, suppose $Q \nsubseteq \mathfrak{p}^{n} M$. Then there exists a positive integer $t$ such that $Q \subseteq \mathfrak{p}^{t} M$ and $Q \nsubseteq \mathfrak{p}^{t+1} M$. Now, just as in the preceding paragraph, we have $Q=\mathfrak{p}^{t} M$.

Let $N$ be a graded submodule of a finitely generated graded $R$-module $M$ and $\mathfrak{p}$ be a minimal prime ideal over $\left(N:_{R} M\right)$. As it was shown in Remark (e), $N(\mathfrak{p})$ is a $\mathfrak{p}$ primary submodule of $M$. By the previous theorem, whenever $M$ is a finitely generated generalised graded multiplication $R$-module, then there exists a positive integer $n$ such that $N(\mathfrak{p})=\mathfrak{p}^{n} M$, that is the isolated $\mathfrak{p}$-primary component of $N$ is just $\mathfrak{p}^{n} M$ for some positive integer $n$.

PROPOSITION 4.7. Let $M$ be a finitely generated generalised graded multiplication $R$-module and $N$ be a graded submodule of $M$. Let $\left\{\mathfrak{p}, \mathfrak{q}_{\lambda}\right\}_{\lambda \in \Lambda}$ be the set of all minimal prime ideals of $\left(N:_{R} M\right)$ and $\mathfrak{p}^{n} M=N(\mathfrak{p})$ be the isolated $\mathfrak{p}$-primary component of $N$. If $\mathfrak{p}^{n} M \neq \mathfrak{p}^{n+1} M$, then

$$
\bigcap_{\lambda \in \Lambda} N\left(\mathfrak{q}_{\lambda}\right) \nsubseteq \mathfrak{p} M
$$

Proof. For each $\lambda \in \Lambda$, let $\mathfrak{q}_{\lambda}^{n_{\lambda}} M$ be the isolated $\mathfrak{q}_{\lambda}$-primary component of $N$. Let $L=\bigcap_{\lambda \in \Lambda} \mathfrak{q}_{\lambda}^{n_{\lambda}} M$. Then by Theorem 3.6, we have $N=\mathfrak{p}^{n} M \bigcap L$. Now by the same argument as in the proof of Theorem 3.6, p $M$ is a graded prime submodule of $M$ and $\left(\mathfrak{p} M:_{R} M\right)=\mathfrak{p}$. Therefore, applying Corollary 4.2 for the graded prime submodule $\mathfrak{p} M, \mathfrak{p}$ should be an *maximal ideal of $R$. By [19, Section 2.13, Lemma 14], this gives that $\mathfrak{p}^{n+1} M$ is a $\mathfrak{p}$-primary submodule of $M$. We have $N \nsubseteq \mathfrak{p}^{n+1} M$, otherwise

$$
\mathfrak{p}^{n} M=N(\mathfrak{p}) \subseteq\left[\mathfrak{p}^{n+1} M\right](\mathfrak{p})=\mathfrak{p}^{n+1} M,
$$

which contradicts the hypothesis. So there exists a graded ideal $\mathfrak{a}$ of $R$ such that $\mathfrak{a} \nsubseteq \mathbb{p}$ and $N=\mathfrak{a p}^{n} M$. Now for each $\lambda \in \Lambda$, we have $\mathfrak{a p}^{n} M=N \subseteq \mathfrak{q}_{\lambda}^{n_{\lambda}} M$ and $\mathfrak{p}^{n} \not \subset \mathfrak{q}_{\lambda}$; thus, $\mathfrak{a} M \subseteq \mathfrak{q}_{\lambda}^{n_{\lambda}} M$ because of $\mathfrak{q}_{\lambda}^{n_{\lambda}} M$ is a $\mathfrak{q}_{\lambda}$-primary submodule. Consequently, $\mathfrak{a} M \subseteq L$, and as by [14, Result 2] $\mathfrak{a} M \nsubseteq \mathfrak{p} M$, we must have $L \nsubseteq \mathfrak{p} M$ as desired.

Combining Theorems 4.3, 4.6 and Proposition 4.7, we can conclude the following characterisation theorem.

THEOREM 4.8. Let $M$ be a finitely generated graded multiplication R-module. The following two statements are equivalent:

(1) $M$ is a generalised graded multiplication R-module.

(2) M satisfies the following conditions:

(i) For each graded submodule $N$ of $M, N=\bigcap_{\mathfrak{q}} N(\mathfrak{q})$, where $\mathfrak{q}$ runs over all minimal prime ideals of $\left(N:_{R} M\right)$.

(ii) For each graded primary submodule $Q$ of $M$, there exists $n \in \mathbb{N}$ such that $Q=\left(\sqrt{Q:_{R} M}\right)^{n} M$.

(iii) If $N$ is a graded submodule of $M$ and $\mathfrak{p}$ is a minimal prime ideal of $\left(N:_{R} M\right)$, if $n$ is the least positive integer such that $\mathfrak{p}^{n} M=N(\mathfrak{p})$ and if $\mathfrak{p}^{n} M \neq \mathfrak{p}^{n+1} M$, then $\bigcap_{\mathfrak{q}} N(\mathfrak{q}) \nsubseteq \mathfrak{p} M$, where $\mathfrak{q}$ runs over all minimal prime ideals of $\left(N:_{R} M\right)$ other than $\mathfrak{p}$. 
ACKNOWLEDGEMENT. I would like to thank the referees for their carefully reading of the paper and offering insightful comments.

\section{REFERENCES}

1. Z. Abd EL-Baset and P. F. Smith, Multiplication modules, Comm. Algebra 16(4) (1988), 755-779.

2. M. Ali and D. Smith, Some remarks on multiplication and projective modules, Comm. Algebra 32 (2004), 3897-3909.

3. D. Anderson and Y. Al-Shaniafi, Multiplication modules and the ideal $\theta(M)$, Comm. Algebra 30 (2002), 3383-3390.

4. A. D. Barnard, Multiplication modules, J. Algebra 70 (1981), 303-315.

5. W. Bruns and J. Herzog, Cohen-Macaulay rings (Cambridge University Press, Cambridge, UK, 1998).

6. SH. Ebrahimi Atani and R. Ebrahimi Atani, Graded multiplication modules and the graded ideal $\theta_{g}(M)$, Turk. J. Math. 33 (2009), 1-9.

7. V. Erdogdu, Multiplication modules which are distributive, J. Pure Appl. Algebra 54 (1988), 209-213.

8. J. Escoriza and B. Torrecillas, Multiplication graded rings, Lect. Notes Pure Appl. Math. 208 (2000), 127-137.

9. J. Escoriza and B. Torrecillas, Multiplication objects in commutative Grothendieck category, Comm. Algebra 26 (1998), 1867-1883.

10. S. Goto and K. Watanabe, On graded rings I, J. Math. Soc. Japan 30 (1978), 172-213.

11. T. W. Hungerford, Algebra (Springer, New York, 1974).

12. W. Krull, Ûber allgemeine Multiplikationringe, Tohoku Math. J. 41 (1935), 320-326.

13. M. D. Larsen and P. J. McCarthy, Multiplicative theory of ideals (Academic Press, Maryland Heights, MO, 1971).

14. R. L. McCasland and M. E. Moore, On radicals of submodules of finitely generated modules, Canad. Math. Bull. 29(1) (1986), 37-39.

15. R. L. McCasland and M. E. Moore, Prime submodules, Comm. Algebra 20(6) (1992), $1803-1817$.

16. R. L. McCasland and P. F. Smith, Prime submodules of Noetherian modules, Rocky Mountain J. Math. 23(3) (1993), 1041-1062. 1986).

17. H. Matsumura, Commutative ring theory (Cambridge University Press, Cambridge, UK,

18. C. Nastasescu and F. Van Oystaeyen, Graded ring theory (North-Holland, Amsterdam, Netherlands, 1982).

19. D. G. Northcott, Lessons on rings, modules and multiplicities (Cambridge University Press, Cambridge, UK, 1968).

20. P. F. Smith, Some remarks on multiplication modules, Arch. Math. 50 (1988), 223-235.

21. A. Tuganbaev, Distributive and multiplication modules and rings, Math. Notes $\mathbf{7 5}$ (2004), 391-400. 